\title{
Poisoning Severity Score as a Predictor of Cardiotoxicity Induced by Anticholinesterase Pesticides, Digoxin and Beta Blockers
}

\author{
Ayman M. Zaaqoq; Hany A. Gamaluddin; Enas A. El-Taftazani; Gamal N. E. Al- \\ Sayed; Samar A. Ahmed'
}

${ }^{1}$ Department of Forensic Medicine and Clinical Toxicology, Faculty of Medicine, Ain Shams University, Cairo, Egypt.

All rights reserved.

\begin{abstract}
Poisoning and fatalities by cardiotoxic agents represent a challenging health problem in Egypt. An important action to combat this problem is to predict or, at least, early diagnose cardiac involvement. To do so, the clinician needs both bedside skills and appropriately selected laboratory testing. The Poisoning Severity Score (PSS) has been evaluated in one study which found it to be useful in identifying serious and complicated cases of poisoning. The aim of this study was to investigate effectiveness of the PSS in predicting cardiotoxicity, as well as correlations of different demographic, exposure, clinical and laboratory findings to cardiotoxicity. Methodology: Over a period of 4 months, we investigated 59 patients with anticholinesterases $(\mathrm{n}=28)$, digoxin $(\mathrm{n}=17)$, and beta-blocker toxicities $(\mathrm{n}=14)$ admitted to Poison Control Center of Ain Shams University Hospitals (PCCA), Cairo, Egypt, in addition to 16 healthy controls. For each, age, sex, mode of exposure, compound involved, time elapsed between exposure and admission, length of hospital stay, clinical, laboratory, and electrocardiographic findings were recorded. Also, PSS was calculated. Results: Female gender, lag between exposure and admission, length of ICU stay, and total length of hospital stay were significantly correlated to the severity of cardiotoxicity. Vomiting, metabolic acidosis, respiratory alkalosis, and PSS were independent predictors of cardiotoxicity. A PSS of 2 had a sensitivity of $88 \%$ and a specificity of $64.7 \%$ in predicating cardiotoxicity. Conclusion: Implication of PSS in prediction and early diagnosis of cardiotoxicity is easy, available, cheap, and reliable, whatever the type of toxic exposure.
\end{abstract}

\section{Introduction}

$\mathrm{M}$ aintenance of adequate tissue perfusion depends on the volume status, vascular resistance, cardiac contractility, and cardiac rhythm. These components of the hemodynamic system are all vulnerable to the effects of xenobiotics. Cardiovascular toxicity may be manifested by the development of hemodynamic instability, dysrhythmias, or heart failure (Hessler, 2006).

Poisoning and fatalities by cardiotoxic agents represent a challenging health problem in Egypt. Reviewing the available studies about magnitude of poisoning in different Egyptian governorates reveal that anticholinesterases come often in the first place as the major cardiotoxic agents, followed by addicting agents, zinc phosphide, and cardiovascular drugs. In Cairo, studies run in the Poison Control Center of Ain Shams University (PCCA) from 2003 to 2007 showed that exposure to cardiotoxic agents represented $37-66 \%$ of the studied cases; and resulted in $40-83 \%$ of total deaths (Abdel-Salam et al., 2005; Gamaluddin, 2005; Gamaluddin et al., 2006; Gamaluddin, 2007). Major agents were anticholinesterases, zinc phosphide, addicting agents, cardiovascular drugs, and scorpion envenomation. In a more recent study, cardiotoxic agents were involved in more than $38 \%$ of 246 acutely poisoned geriatric patients presented to the PCCA during 2010, and resulted in $83 \%$ of geriatric deaths that year. Organophosphates affected 24 cases $(9.8 \%)$, followed by benzodiazepines $(6.5 \%)$, cardiovascular drugs and antidepressants ( $4 \%$ each). Cardiovascular affection was the commonest cause of ICU admission; either alone or in combination with other system involvement. Out of 37 ICU admitted patients, twenty (54\%) had severe 
hypotension, bradycardia and/or heart block (Ebrahim et al., 2011).

An important action to combat this problem is to predict or, at least, early diagnose cardiac involvement. To do so, the clinician needs both bedside skills and appropriately selected laboratory testing (Grais, 2010). Hoefman and colleagues (2007) concluded that prediction of arrhythmias by general practitioners (GPs) based on history taking and physical examination alone is not accurate. They stressed on the importance of adding new diagnostic facilities for an adequate diagnostic process

A number of systems have been proposed for predicting outcome in OP poisoning, many rely on laboratory tests (Eddleston et al., 2005).

The Poisoning Severity Score (PSS) was developed by the International Program on Chemical Safety (IPCS), the European Community (EC), and the European Association of Poisons Centers and Clinical Toxicologists (EAPCCT) to create a scoring system that produces a qualitative evaluation of the morbidity caused by different forms of poisoning (Persson et al., 1998). This classification scheme is used for acute poisonings in both adults and children regardless of the type and number of agents involved. It has several different categories which encompass a large number of clinical features and it is designed to be used flexibly to incorporate the most relevant clinical and laboratory features of the poisoning and data available (Table 1). This has been evaluated prospectively in one study which found it to be useful in identifying serious and complicated cases of poisoning (Casey et al., 1998).

The aim of this study was to investigate effectiveness of the PSS in predicting acute cardiotoxicity, as well as correlations of different demographic, exposure, clinical and laboratory findings to cardiotoxicity.

\section{Methodology}

\section{Design and setting}

The present study is a nested case-control study, performed on selected patients admitted to the Poison Control Center of Ain Shams University Hospitals (PCCA), Cairo, Egypt; with exposure to anticholinesterase pesticides, digoxin, or beta blockers; during the period from August to November 2011.

The PCCA was instituted in December 1981 as the first of its kind in Egypt, providing integrated services in diagnosis and treatment of acute intoxication. It gives help to more than twenty thousand patients per year (Gamaluddin et al., 2006; Gamaluddin, 2007; Ebrahim et al., 2011).

\section{Patient selection criteria}

A written informed consent was obtained from each studied individual or the person on his/her behalf. The diagnosis of intoxications was based on history of exposure within $6 \mathrm{~h}$ to an anticholinesterase pesticide, digoxin or a beta blocker; in addition to physical examination and/or laboratory detection.

The choice of anticholinesterase pesticides was made due to the large number of cases presented (nearly $43 \%$ of all cardiotoxicity cases according to Gamaluddin (2007)). Digoxin and beta blockers have been chosen because both cause cardiovascular disturbances at high rates (Hack \& Lewin, 2006; Sharma et al., 2011). Besides, the selected agents act differently (Kang, 2008).

The diagnosis of acute cardiotoxicity was anchored in the presence of one or more of the clinical manifestations that include hemodynamic instability, heart failure, cardiac conduction abnormalities and dysrhythmias (Hessler, 2006); and/or ECG manifestations. Patients with past history of cardiac disease in addition to those older than 60 years were eliminated from the study.

\section{Groups and sampling}

In the current study, stratified sampling was applied to select individuals of both sexes, divided into 3 groups:

- Group 1 - Control: healthy, nonexposed persons.

- Group 2 - Exposure without toxicity: patients exposed to one of the selected cardiotoxic agents, but without detectable cardiotoxicity, subdivided according to the type of toxic agent.

- Group 3-Overt cardiotoxicity: acutely poisoned patients showing manifestations of cardiotoxicity, subdivided according to the type of cardiotoxic exposure.

\section{Parameters}

For each of the selected patients, clinical and investigational parameters were carefully probed and evaluated. The choice of these parameters was decided because they were used to calculate the PSS; in addition, they were common between the selected agents. They included:

\section{A-History}

History was obtained from the poisoned patient, his/her relatives, friends, pre-hospital personnel, the patient's physician or therapist. It included personal history, history of present poisoning, and cardiovascular history.

\section{B- Physical examination}

Vital signs were assessed on admission and then hourly for patients admitted to the ICU and every $2 \mathrm{~h}$ for patients admitted to the inpatient ward until discharge. Various body systems were examined on admission, $6 \mathrm{~h}$ after, and then every $12 \mathrm{~h}$ for ICU patients and every $24 \mathrm{~h}$ for those in the inpatient ward until discharge.

\section{C-Investigations}

For each patient, the following was done at the time of admission, $6 \mathrm{~h}$ after and then every $24 \mathrm{~h}$ unless otherwise indicated. 


\section{Electrocardiography (ECG)}

In the present thesis, rate-corrected QT (QTc) was evaluated using Hodge's formula (Luo et al., 2004), in which:

$$
Q T c=Q T+1.75 \text { (heart rate }-60)
$$

Where QTc and QT interval are measured in milliseconds, heart rate in beat/minute. The upper normal limit of QTc determined by this formula was $457 \mathrm{~ms}$ for both sexes.

\section{Arterial blood gases (ABG)}

Each blood sample for the analysis of $A B G$, electrolytes, and hemoglobin consisted of 1-2 mL of arterial blood withdrawn from either radial or femoral artery by a heparinized syringe.

\section{Serum sodium and potassium}

\section{Hemoglobin was measured in the context}

\section{of ABG analysis.}

\section{D-Prognosis}

The prognosis of cases was evaluated according to the PSS (Table 1), severity of cardiotoxic manifestations (according to Persson et al., (1998)), length of hospital stay (in intensive care unit (ICU), inpatient ward (IP), in addition to their sum in hours), and final outcome. Occurrence of a particular symptom was checked against the chart and graded. The severity grading assigned to a case was determined by the most severe symptom(s) or signs(s) observed. The severity was graded from 0 to 4 , ranging from no toxicity to severe life threatening symptoms and death and taking into consideration clinical signs/symptoms and/or laboratory data. Before discharge, the patient's whole clinical course was revised to determine the highest PSS.

\section{Statistical analysis}

Data was statistically analyzed using SPSS $^{\circledR} 17.0$ (IBM, 2008; New York, USA), Analyse-it ${ }^{\mathbb{B}}$ add in for Excel (Analyse-it Software Ltd., 2008; Leeds, UK), and Microsoft $^{\circledR}$ Excel $^{\mathrm{TM}} 2007$ (Microsoft corporation, 2007; Redmond, USA).

Descriptive and inferential statistics were done after assessing normality of data distribution. Inferential statistics included tests of significance, regression analysis, and Receiver Operating Characteristic (ROC) curve.

Table 1: Poisoning Severity Score (modified from Persson et al., (1998)).

\begin{tabular}{|c|c|c|c|}
\hline \multirow[b]{3}{*}{ Organ } & \multicolumn{3}{|c|}{ Poisoning Severity Score ${ }^{a}$} \\
\hline & 1 & 2 & 3 \\
\hline & $\begin{array}{c}\text { Minor } \\
\text { Mild, transient, and } \\
\text { spontaneously resolving } \\
\text { symptoms or signs }\end{array}$ & $\begin{array}{c}\text { Moderate } \\
\text { Pronounced or prolonged symptoms } \\
\text { or signs }\end{array}$ & $\begin{array}{c}\text { Severe } \\
\text { Severe or life-threatening symptoms or } \\
\text { signs }\end{array}$ \\
\hline GI tract & $\begin{array}{l}\text {-Vomiting, diarrhea, } \\
\text { pain }\end{array}$ & $\begin{array}{l}\text {-Pronounced or prolonged } \\
\text { vomiting, diarrhea, pain } \\
\text { ileus }\end{array}$ & $\begin{array}{l}\text { - Massive hemorrhage, } \\
\text { perforation }\end{array}$ \\
\hline $\begin{array}{l}\text { Respiratory } \\
\text { system }\end{array}$ & $\begin{array}{l}\text { - Irritation, coughing, } \\
\text { breathlessness, mild } \\
\text { dyspnea, mild } \\
\text { bronchospasm } \\
\text { - Chest X ray: Abnormal } \\
\text { with minor or no } \\
\text { symptoms }\end{array}$ & $\begin{array}{l}\text { - Prolonged coughing, } \\
\text { bronchospasm, dyspnea, } \\
\text { stridor, hypoxemia requiring } \\
\text { extra oxygen } \\
\text { - Chest X ray: Abnormal with } \\
\text { moderate symptoms }\end{array}$ & $\begin{array}{l}\text { - Manifest respiratory } \\
\text { insufficiency (e.g., severe } \\
\text { bronchospasm, airway } \\
\text { obstruction, glottal edema, } \\
\text { pulmonary edema, ARDS } \\
\text { pneumonitis, pneumonia, } \\
\text { pneumothorax) } \\
\text {-Chest X ray: Abnormal with } \\
\text { severe symptoms }\end{array}$ \\
\hline $\begin{array}{l}\text { Nervous } \\
\text { system }\end{array}$ & $\begin{array}{l}\text { - Drowsiness, vertigo, } \\
\text { tinnitus, ataxia } \\
\text { - Restlessness } \\
\text { - Mild cholinergic } \\
\text { /anticholinergic } \\
\text { symptoms }\end{array}$ & $\begin{array}{l}\text { - Unconsciousness with } \\
\text { appropriate response to pain } \\
\text { - Brief apnea, bradypnea } \\
\text { - Confusion, agitation, } \\
\text { hallucinations, delirium } \\
\text { - Infrequent, generalized, or } \\
\text { local seizures } \\
\text { - Pronounced cholinergic } \\
\text { /anticholinergic symptoms }\end{array}$ & $\begin{array}{l}\text { - Deep coma with inappropriate } \\
\text { response to pain or unresponsive } \\
\text { to pain } \\
\text { - Respiratory depression with } \\
\text { insufficiency } \\
\text { - Extreme agitation } \\
\text { - Frequent, generalized seizures, } \\
\text { status epilepticus, opisthotonos }\end{array}$ \\
\hline $\begin{array}{l}\text { Cardiovascular } \\
\text { system }\end{array}$ & $\begin{array}{l}\text { - Isolated extrasystoles } \\
\text { - Mild and transient } \\
\text { hypo/hypertension }\end{array}$ & $\begin{array}{l}\text { - Sinus bradycardia } \\
\text { (HRc } 40-50 \text { in adults, } 60-80 \\
\text { in infants and children, } 80- \\
90 \text { in neonates) } \\
\text { - Sinus tachycardia } \\
\text { (HR } 140-180 \text { in adults, } 160- \\
190 \text { in infants and children, } \\
160-200 \text { in neonates) } \\
\text { - Frequent extrasystoles, }\end{array}$ & $\begin{array}{l}\text { - Severe sinus bradycardia } \\
\text { (HR }<40 \text {, in adults, }<60 \text { in } \\
\text { infants, }<80 \text { in neonates) } \\
\text { - Severe sinus tachycardia } \\
\text { (HR }>180 \text { in adults, }>190 \text { in } \\
\text { infants and children, }>200 \text { in } \\
\text { neonates) } \\
\text { - Life-threatening ventricular } \\
\text { dysrhythmias, AV block III, }\end{array}$ \\
\hline
\end{tabular}




\begin{tabular}{|c|c|c|c|}
\hline \multirow[b]{3}{*}{ Organ } & \multicolumn{3}{|c|}{ Poisoning Severity Score ${ }^{a}$} \\
\hline & 1 & 2 & 3 \\
\hline & $\begin{array}{c}\text { Minor } \\
\text { Mild, transient, and } \\
\text { spontaneously resolving } \\
\text { symptoms or signs }\end{array}$ & $\begin{array}{c}\text { Moderate } \\
\text { Pronounced or prolonged symptoms } \\
\text { or signs }\end{array}$ & $\begin{array}{c}\text { Severe } \\
\text { Severe or life-threatening symptoms or } \\
\text { signs }\end{array}$ \\
\hline & & $\begin{array}{l}\text { atrial fibrillation/flutter, } \\
\text { AVd block I -II, prolonged } \\
\text { QRS and QTc time, } \\
\text { repolarization abnormalities } \\
\text { - Myocardial ischemia } \\
\text { - More pronounced } \\
\text { hypo/hypertension }\end{array}$ & $\begin{array}{l}\text { asystole } \\
\text { - Myocardial infarction } \\
\text { - Shock, hypertensive crisis }\end{array}$ \\
\hline $\begin{array}{l}\text { Metabolic } \\
\text { balance }\end{array}$ & $\begin{array}{l}\text { - Mild acid-base } \\
\text { disturbances (HCO3- } \\
\sim 15-20 \text { or } 30-40 \\
\mathrm{mEq} / \mathrm{L}, \mathrm{pH} \sim 7.25-7.32 \\
\text { or } 7.5-7.59) \\
\text { - Mild electrolyte and } \\
\text { fluid disturbances }(\mathrm{K}+ \\
3-3.4 \text { or } 5.2-5.9 \mathrm{mEq} / \mathrm{L}) \\
\text { - Mild hypoglycemia } \\
\text { (50-70 mg/dL) } \\
\text { - Hyperthermia of short } \\
\text { duration }\end{array}$ & $\begin{array}{l}\text { - More pronounced acid- } \\
\text { base disturbances (HCO3- } \\
\sim 10-14 \text { or }>40 \mathrm{mEq} / \mathrm{L}, \\
\mathrm{pH} 7.15-7.24 \text { or } 7.6-7.69) \\
\text { - More pronounced } \\
\text { electrolyte and fluid } \\
\text { disturbances }(\mathrm{K}+2.5-2.9 \text { or } \\
6-6.9 \mathrm{mEq} / \mathrm{L}) \\
\text { - More pronounced } \\
\text { hypoglycemia (30-50 } \\
\text { mg/dL) } \\
\text { - Hyperthermia of longer } \\
\text { duration }\end{array}$ & $\begin{array}{l}\text { - Severe acid-base disturbances } \\
(\mathrm{HCO} 3-<10 \mathrm{mEq} / \mathrm{L}, \mathrm{pH}<7,15 \\
\text { or }>7.7) \\
\text { - Severe electrolyte and fluid } \\
\text { disturbances }(\mathrm{K}+<2.5 \text { or }>7 \\
\mathrm{mEq} / \mathrm{L}) \\
\text { - Severe hypoglycemia }(<30 \\
\mathrm{mg} / \mathrm{dL}) \\
\text { - Dangerous hypo/hyperthermia }\end{array}$ \\
\hline Blood & & $\begin{array}{l}\text { Anemia, leucopenia, } \\
\text { thrombocytopenia }\end{array}$ & $\begin{array}{l}\text { - Severe anemia, leucopenia, } \\
\text { thrombocytopenia }\end{array}$ \\
\hline $\begin{array}{l}\text { Muscular } \\
\text { system }\end{array}$ & $\begin{array}{l}\text { - Mild pain, tenderness } \\
\text { - } \mathrm{CKe} \sim 250-1500 \mathrm{IU} / \mathrm{L}\end{array}$ & $\begin{array}{l}\text { Pain, rigidity, cramping, } \\
\text { and fasciculations } \\
\text { - Rhabdomyolys, CK 1500- } \\
10000 \mathrm{IU} / \mathrm{L}\end{array}$ & $\begin{array}{l}\text { - Intense pain, extreme rigidity, } \\
\text { extensive cramping, and } \\
\text { fasciculations } \\
\text { - Rhabdomyolysis with } \\
\text { complications or compartment } \\
\text { syndrome, } \mathrm{CK} \geq 10000 \mathrm{IU} / \mathrm{L}\end{array}$ \\
\hline $\begin{array}{l}{ }^{c} H R, \text { hea } \\
{ }^{d} A V \text {, atri }\end{array}$ & $\begin{array}{l}\text { ades are not included in } \\
\text { espiratory Distress Synd } \\
\text { icular } \\
\text { nase }\end{array}$ & le: 0 (none: no symptoms or sigr & and 4 (fatal: death). \\
\hline
\end{tabular}

\section{Results}

The current study was conducted on 75 individuals of both sexes. Their demographic and exposure characteristics are shown in Table 2. The patients under study were classified according to their ages into 3 groups: preschool age group $(<6 \mathrm{y})$, school age group (6$18 \mathrm{y}$ ), and legal adulthood group ( $>18 \mathrm{y}$ according to the definition adopted by the Egyptian Child Law, (2008)). Table 3 shows that accidental exposure was significantly less common in school and adult groups compared to preschool group, in contrary to suicidal mode of exposure which was significantly more common in school and adult groups compared to preschool group. In addition, no significant difference existed between age groups according to the type of exposure.

As regards gender, Table 4 demonstrates that cardiotoxicity was significantly higher in females compared to males (Figure 2). In addition, lag between exposure and admission had statistically significant negative correlation with the degree of cardiac toxicity (Figure 3). Likewise, vomiting was significantly more prominent in cardiotoxicity group (Table 5). On the other hand, all other extracardiac manifestations showed nonsignificant differences between non- and cardiotoxic patients. Similarly, there were no statistically significant differences in all laboratory parameters between patients with cardiotoxicity and those without cardiotoxicity except for metabolic acidosis and respiratory alkalosis, both of which were significantly more distinct in cardiotoxic patients (Table 6). Regression analysis showed that vomiting, metabolic acidosis, and respiratory alkalosis were independent predictors of cardiotoxicity (Table 10).

Concerning ECG findings of the studied patients (Table 7), the commonest reported degree of heart block was the second one $(n=6,10 \%)$, followed by the first degree $(\mathrm{n}=5,8.5 \%)$, and only one patient had complete heart block. Only two cases demonstrated premature beats and one had wide QRS. Prolonged QTc ensued in five patients $(8.5 \%)$. Among group of overt 
cardiotoxicity, three had mild degree of cardiac affection, 18 had moderate, and four had severe cardiac affection.

As to the length of hospital stay, a highly significant positive correlation was noticed between the length of ICU/total hospital stay and degree of cardiotoxicity (Table 8). In respect of final outcome, the vast majority of the selected patients recovered completely $(n=58,98.3 \%)$, and only one patient with suicidal anticholinesterase poisoning died. This patient was a 40 years old male who was presented to the PCCA an hour after exposure. On admission, he had a PSS of 3. The patient developed severe cardiotoxicity in the form of severe hypotension, $3^{\text {rd }}$ degree heart block, and prolonged QTc. He also developed respiratory failure and required mechanical ventilation. The patient demonstrated severe mixed acidosis and moderate hypokalemia; and died $40 \mathrm{~h}$ after admission to the ICU.

On the topic of PSS, no statistically significant difference existed between the causative toxins (Table 9 and Figure 1). Regression analysis showed that PSS is an independent predictor of cardiotoxicity (Table 10). ROC analysis showed that PSS $0-1$ had highest sensitivity but with minimal specificity. Score 2 was the cut-off point for a "general optimum test" with a sensitivity of $88 \%$ and a specificity of $64.7 \%$ (Table 11 and Figure 4 ).

Table 2: Demographic and exposure characteristics of the studied individuals.

\begin{tabular}{|c|c|c|c|c|c|c|c|c|c|}
\hline \multirow{2}{*}{\multicolumn{2}{|c|}{ Description }} & \multicolumn{2}{|c|}{\begin{tabular}{|c|} 
Group \\
1 \\
Control \\
$(n=16)$ \\
\end{tabular}} & \multicolumn{2}{|c|}{$\begin{array}{c}\text { Group } 2 \\
\text { Exposure without cardio- } \\
\text { toxicity } \\
(n=34)\end{array}$} & \multicolumn{2}{|c|}{$\begin{array}{c}\text { Group } 3 \\
\text { Overt cardio- } \\
\text { toxicity } \\
(n=25) \\
\end{array}$} & \multicolumn{2}{|c|}{$\begin{array}{c}\text { Groups } \\
2+3 \\
\text { All cases } \\
(\mathbf{n}=\mathbf{5 9})\end{array}$} \\
\hline & & n & $\%$ & $\mathbf{n}$ & $\%$ & $\mathbf{n}$ & $\%$ & $\mathbf{n}$ & $\%$ \\
\hline \multirow[t]{2}{*}{ Gender } & Male $(n=25)$ & 10 & 62.5 & 12 & 35.3 & 3 & 12 & 15 & 25.4 \\
\hline & Female $(n=50)$ & 6 & 37.5 & 22 & 64.7 & 22 & 88 & 44 & 74.6 \\
\hline \multirow[t]{3}{*}{ Agent } & Anti-cholinesterase & 0 & 0 & 18 & 53 & 10 & 40 & 28 & 47.5 \\
\hline & Digoxin & 0 & 0 & 8 & 23.5 & 9 & 36 & 17 & 28.8 \\
\hline & Beta-blockers & 0 & 0 & 8 & 23.5 & 6 & 24 & 14 & 23.7 \\
\hline \multirow[t]{2}{*}{ Mode of exposure } & Suicidal & 0 & 0 & 25 & 73.5 & 18 & 72 & 43 & 72.9 \\
\hline & Accidental & 0 & 0 & 9 & 25.5 & 7 & 28 & 16 & 27.1 \\
\hline \multirow{4}{*}{$\begin{array}{l}\text { Time between exposure } \\
\text { and admission (hours) }\end{array}$} & Minimum & & 0 & \multicolumn{2}{|c|}{1} & \multicolumn{2}{|c|}{0.5} & \multicolumn{2}{|c|}{0.5} \\
\hline & Maximum & & 0 & \multicolumn{2}{|c|}{6} & \multicolumn{2}{|c|}{6} & \multicolumn{2}{|c|}{6} \\
\hline & Mean & & 0 & \multicolumn{2}{|c|}{3.57} & \multicolumn{2}{|c|}{2.7} & \multicolumn{2}{|c|}{3.22} \\
\hline & SD & & 0 & \multicolumn{2}{|c|}{1.88} & \multicolumn{2}{|c|}{2} & \multicolumn{2}{|c|}{1.97} \\
\hline
\end{tabular}

${ }^{a} P$ is significant if $<.05$ using Kruskal-Wallis test.

Table 3: Distribution of patients according to their ages.

\begin{tabular}{|c|c|c|c|c|c|}
\hline \multicolumn{3}{|c|}{ Variable } & Preschool $(<6$ y) & School (6-18 y) & Legal adulthood $^{\mathrm{a}}(>18 \mathrm{y})$ \\
\hline \multirow{5}{*}{$\begin{array}{l}\text { Age for } \\
\text { all } \\
\text { patients } \\
\text { (years) }\end{array}$} & \multicolumn{2}{|c|}{ Number $/$ Percent $(n=59)$} & $8(13.6 \%)$ & $17(28.8 \%)$ & $34(57.6 \%)$ \\
\hline & \multicolumn{2}{|l|}{ Minimum } & 1.5 & 9 & 20 \\
\hline & \multicolumn{2}{|l|}{ Maximum } & 5 & 18 & 54 \\
\hline & \multicolumn{2}{|l|}{ Mean } & 2.63 & 14.53 & 29.6 \\
\hline & \multicolumn{2}{|l|}{ SD } & 1.1 & 3.1 & 9.7 \\
\hline \multirow[t]{4}{*}{ Agent } & \multicolumn{2}{|c|}{ Anticholinesterases $(n=28)$} & $5(18 \%)$ & $9(32 \%)$ & $14(50 \%)$ \\
\hline & \multicolumn{2}{|l|}{ Digoxin $(n=17)$} & $2(11.8 \%)$ & $3(17.6 \%)$ & $12(70.6 \%)$ \\
\hline & \multicolumn{2}{|c|}{ Beta blocker $(n=14)$} & $1(7 \%)$ & $5(35.7 \%)$ & $8(57 \%)$ \\
\hline & \multicolumn{2}{|c|}{ P (Kruskal-Wallis test) } & \multicolumn{3}{|c|}{$.58(\mathrm{NS})$} \\
\hline \multirow{6}{*}{$\begin{array}{l}\text { Mode of } \\
\text { exposure }\end{array}$} & \multicolumn{2}{|l|}{ Suicidal } & 0 & $13(76.5 \%)$ & $30(88.2 \%)$ \\
\hline & \multicolumn{2}{|c|}{ Accidental } & $8(100 \%)$ & $4(23.5 \%)$ & $4(11.8 \%)$ \\
\hline & \multicolumn{2}{|c|}{ P (Kruskal-Wallis test) } & \multicolumn{3}{|c|}{$.000(\mathrm{HS})$} \\
\hline & \multirow{3}{*}{$\begin{array}{l}\text { Contrast (LSD } \\
\text { test) }\end{array}$} & vs. Preschool & - & $.000(\mathrm{HS})$ & $.000(\mathrm{HS})$ \\
\hline & & Vs. School & $.000(\mathrm{HS})$ & - & $.25(\mathrm{NS})$ \\
\hline & & vs. Adult & $.000(\mathrm{HS})$ & $.25(\mathrm{NS})$ & - \\
\hline
\end{tabular}

\footnotetext{
${ }^{a}$ According to the Egyptian Child Law, (2008).
}

Table 4: Correlations of demographic and exposure characteristics to cardiotoxicity in the studied cases.

\begin{tabular}{|l|l|l|l|l|l|l|} 
Variable & $\mathbf{N}$ & Mean & SD & Test & $\mathbf{P}^{\text {a }}$ & Sig. \\
\hline
\end{tabular}




\begin{tabular}{|c|c|c|c|c|c|c|c|}
\hline \multirow[t]{2}{*}{ Gender } & Male & 15 & .4 & .9 & \multirow{2}{*}{ Mann-Whitney test, $Z=-1.97$} & \multirow[t]{2}{*}{.049} & \multirow[t]{2}{*}{$\mathrm{S}$} \\
\hline & Female & 44 & 1 & 1.1 & & & \\
\hline \multirow[t]{2}{*}{ Mode of exposure } & Suicidal & 43 & .86 & .16 & \multirow[t]{2}{*}{ Mann-Whitney test, $\mathrm{Z}=-.08$} & \multirow[t]{2}{*}{.94} & \multirow[t]{2}{*}{ NS } \\
\hline & Accidental & 16 & .88 & .29 & & & \\
\hline \multirow[t]{3}{*}{ Age } & Preschool & 8 & 1.13 & 1.36 & \multirow{3}{*}{ Kruskal-Wallis test, Kruskal-Wallis' statistic $=3.4$} & \multirow[t]{3}{*}{.18} & \multirow[t]{3}{*}{ NS } \\
\hline & School & 17 & 1.18 & 1.07 & & & \\
\hline & Adult & 34 & .65 & .98 & & & \\
\hline \multicolumn{2}{|c|}{ Time between exposure and admission } & 59 & .9 & 1.1 & Spearman's test, Spearman's rho $=-.28$ & .034 & $\mathrm{~S}$ \\
\hline
\end{tabular}

${ }^{a} P$ is significant if $\leq .05$, and highly significant if $\leq .001$

Table 5: Clinical observations in the studied groups, with Mann-Whitney comparison of extracardiac manifestations in between them.

\begin{tabular}{|c|c|c|c|c|c|c|c|c|c|}
\hline \multirow{2}{*}{\multicolumn{2}{|c|}{ Variable }} & \multicolumn{2}{|c|}{\begin{tabular}{|c|} 
Group 2 \\
Exposure without cardiotoxicity $(\mathrm{n}=34)$ \\
\end{tabular}} & \multicolumn{2}{|c|}{\begin{tabular}{|c|} 
Group 3 \\
Overt cardiotoxicity $(\mathrm{n}=\mathbf{2 5})$ \\
\end{tabular}} & \multicolumn{2}{|c|}{$\begin{array}{c}\text { Groups } 2+3 \\
\text { All cases }(n=59)\end{array}$} & \multirow[t]{2}{*}{$\mathbf{P}^{\mathrm{g}}$} & \multirow[t]{2}{*}{ Sig. } \\
\hline & & \multirow{2}{*}{$\begin{array}{c}\text { Frequency } \\
22\end{array}$} & \multirow{2}{*}{$\begin{array}{c}\text { Percent } \\
64.7\end{array}$} & \multirow{2}{*}{$\begin{array}{c}\text { Frequency } \\
10\end{array}$} & \multirow{2}{*}{\begin{tabular}{c|} 
Percent \\
40
\end{tabular}} & \multirow{2}{*}{\begin{tabular}{|c|} 
Frequency \\
32 \\
\end{tabular}} & \multirow{2}{*}{\begin{tabular}{|c|} 
Percent \\
54.2
\end{tabular}} & & \\
\hline Vomiting $^{\text {a }}$ & Mild & & & & & & & \multirow[t]{3}{*}{.01} & \multirow[t]{3}{*}{$\mathrm{S}$} \\
\hline & Moderate/severe & 3 & 8.8 & 11 & 44 & 14 & 23.7 & & \\
\hline & Total & 25 & 73.5 & 21 & 84 & 46 & 78 & & \\
\hline \multicolumn{2}{|l|}{ Dizziness } & 13 & 38.2 & 16 & 64 & 29 & 49.2 & .052 & $\mathrm{NS}$ \\
\hline \multicolumn{2}{|l|}{ Tachypnea $^{\mathrm{b}}$} & 20 & 58.8 & 17 & 68 & 37 & 62.7 & .48 & $\mathrm{NS}$ \\
\hline \multicolumn{2}{|c|}{ Mechanical ventilation } & 1 & 2.9 & 2 & 8 & 3 & 5.1 & .39 & $\mathrm{NS}$ \\
\hline \multicolumn{2}{|c|}{ Fever $^{\mathrm{c}}$} & 3 & 8.8 & 2 & 8 & 5 & 8.5 & .9 & $\mathrm{NS}$ \\
\hline \multirow[t]{3}{*}{ Bradycardia $^{\mathrm{d}}$} & Mild & 0 & 0 & 4 & 16 & 4 & 6.8 & & \\
\hline & Moderate & 0 & 0 & 10 & 40 & 10 & 16.9 & & \\
\hline & Total & 0 & 0 & 14 & 56 & 14 & 23.7 & & \\
\hline \multirow[t]{3}{*}{ Tachycardia ${ }^{\mathrm{e}}$} & Mild & 0 & 0 & 6 & 24 & 6 & 10.2 & & \\
\hline & Moderate & 0 & 0 & 3 & 12 & 3 & 5.1 & & \\
\hline & Total & 0 & 0 & 9 & 36 & 9 & 15.3 & & \\
\hline \multicolumn{2}{|c|}{ Hypotension $^{\mathrm{f}}$} & 0 & 0 & 6 & 24 & 6 & 10.2 & & \\
\hline \multirow{2}{*}{\multicolumn{10}{|c|}{$\begin{array}{l}\text { Vomiting was rated mild (1-2 times/day), moderate }(3-7 \text { times/day), and severe }(\geq 8 \text { times/day) }(\text { Schmitt, 2010). } \\
{ }^{b} \text { Tachypnea was defined as an increased respiratory rate above normal values for age. The normal rate of respiration in a relaxed adult } \\
\text { is about } 14-16 \text { breath/minute (Moore-Gillon, 2007). Normal rates in children are 20-30 breath/minute (Hartman \& Cheifetz, 2011). } \\
{ }^{c} \text { Fever was defined as a morning temperature } \geq 37.3^{\circ} \mathrm{C} \text { (mouth) or }>37.7^{\circ} \mathrm{C} \text { (rectum) (Magaziner, 2007). }\end{array}$}} \\
\hline & & & & & & & & & \\
\hline \multicolumn{10}{|c|}{$\begin{array}{l}\text { Mild bradycardia at } 50-60 \mathrm{bpm} \text { in adults and } 80-100 \mathrm{bpm} \text { in children; moderate at } 40-50 \mathrm{bpm} \text { in adults and } 60-80 \mathrm{bpm} \text { in children; } \\
\text { severe at rate }<40 \mathrm{bpm} \text { in adults and }<60 \mathrm{bpm} \text { in children (Persson et al., 1998). The current study did not record severe bradycardia. } \\
{ }^{e} \text { Mild tachycardia at } 100-140 \mathrm{bpm} \text { in adults and } 110-160 \mathrm{bpm} \text { in children; moderate at } 140-180 \mathrm{bpm} \text { in adults and } 160-190 \mathrm{bpm} \text { in } \\
\text { children; severe at rate }>180 \mathrm{bpm} \text { in adults and }>190 \mathrm{bpm} \text { in children (Persson et al., 1998). The current study did not record severe } \\
\text { tachycardia. }\end{array}$} \\
\hline \multicolumn{10}{|c|}{$\begin{array}{l}{ }^{f} \text { Hypotension in adults was defined as systolic blood pressure }<100 \mathrm{mmHg} \text { (Jones et al., 2004), while in children, it was considered when } \\
\text { the systolic blood pressure was lower than } 55-70 \mathrm{mmHg} \text { (Hartman \& Cheifetz, 2011). } \\
{ }^{g} P \text { is significant if }<.05\end{array}$} \\
\hline
\end{tabular}

Table 6: Comparison of laboratory findings in the studied groups (Mann-Whitney test).

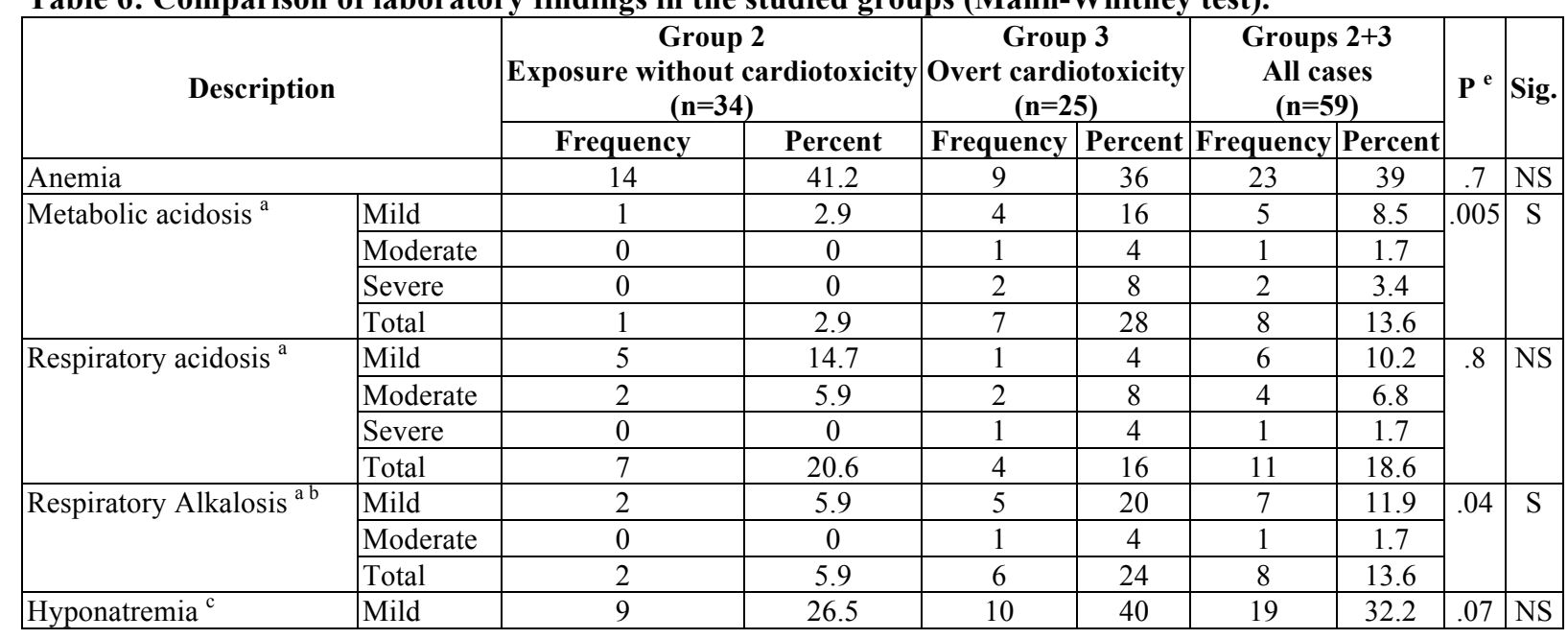




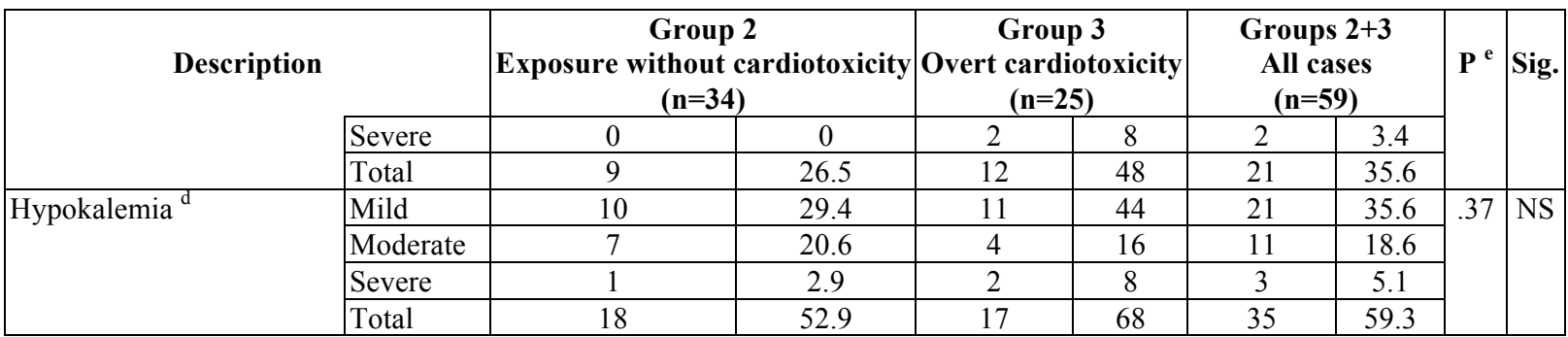

${ }^{a}$ The severity of acid-base disturbances was assessed according to Persson et al. (1998), who classified them into mild (pH 7.25-7.32 or 7.5-7.59; and/or $\mathrm{HCO}_{3} \sim 15-20$ or 30-40 mEq/L), moderate (pH 7.15-7.24 or 7.6-7.69; and/or HCO $10-14$ or $>40 \mathrm{mEq} / \mathrm{L}$ ), and severe disturbances ( $\mathrm{pH}<7.15$ or $\geq 7.7$; and/or $\left.\mathrm{HCO}_{3}<10 \mathrm{mEq} / \mathrm{L}\right)$.

${ }^{b}$ None of patients had metabolic alkalosis.

${ }^{c}$ Hyponatremia was defined as serum $\mathrm{Na}<135 \mathrm{mEq} / \mathrm{L}$, and severe if $<125 \mathrm{mEq} / \mathrm{L}$ (Simon et al., 2012).

${ }^{d}$ Hypokalemia was defined as serum $K<3.5 \mathrm{mEq} / \mathrm{L}$, moderate at $2.5-3 \mathrm{mEq} / \mathrm{L}$, and severe if $<2.5 \mathrm{mEq} / \mathrm{L}(\mathrm{Garth}, 2010)$.

${ }^{e} P$ is significant if $\leq .05$, and highly significant if $\leq .001$

Table 7: ECG observations and severity of cardiotoxicity in the studied series.

\begin{tabular}{|c|c|c|c|c|}
\hline \multicolumn{2}{|c|}{ Description } & Frequency & $\begin{array}{c}\text { Percentage to Group } 3 \\
\text { Overt cardiotoxicity }(n=25)\end{array}$ & $\begin{array}{c}\text { Percentage to Groups } 2+3 \\
\text { All cases }(n=59)\end{array}$ \\
\hline \multirow[t]{4}{*}{ Heart block } & HB I & 5 & 20 & 8.5 \\
\hline & HB II & 6 & 24 & 10.2 \\
\hline & HB III & 1 & 4 & 1.7 \\
\hline & Total & 12 & 48 & 20.3 \\
\hline \multicolumn{2}{|l|}{ Premature beats } & 2 & 8 & 3.4 \\
\hline \multicolumn{2}{|l|}{ Prolonged QTc $^{\mathrm{a}}$} & 5 & 20 & 8.5 \\
\hline \multicolumn{2}{|l|}{ Wide QRS $^{\text {b }}$} & 1 & 4 & 1.7 \\
\hline \multirow[t]{4}{*}{ Cardiotoxicity $^{c}$} & Mild & 3 & 12 & 5.1 \\
\hline & Moderate & 18 & 72 & 30.5 \\
\hline & Severe & 4 & 16 & 6.8 \\
\hline & Total & 25 & 100 & 42.4 \\
\hline
\end{tabular}

${ }^{a}$ QTc was evaluated automatically by the ECG machine then confirmed by manual calculation using Hodge's formula. The upper normal limit of QTc determined by this formula was $457 \mathrm{~ms}$ for both sexes (Luo et al., 2004).

${ }^{b}$ QRS width > 0.1 second (Goldberger, 2006).

${ }^{c}$ According to the PSS (Persson et al., 1998).

Table 8: Correlation of length of hospital stay to the degree of cardiotoxicity (Spearman's test).

\begin{tabular}{|c|c|c|c|c|c|}
\hline \multirow{2}{*}{ Hospital stay ${ }^{a}$} & \multirow{2}{*}{$\begin{array}{c}\text { Mean } \\
\text { (hours) }\end{array}$} & \multirow{2}{*}{ SD } & \multicolumn{3}{|c|}{ Correlation to cardiotoxicity } \\
\hline & & & Spearman's rho & $\mathbf{P}^{\mathbf{b}}$ & Significance \\
\hline $\mathrm{ICU}$ & 20.9 & 15.8 & .46 & .0003 & HS \\
\hline IP & 15.2 & 7.9 & -.05 & .71 & NS \\
\hline Total & 36.1 & 17.1 & .51 & $<.0001$ & HS \\
\hline
\end{tabular}

${ }^{a}$ ICU, intensive care unit; IP, inpatient ward

${ }^{b} P$ is significant if $\leq .05$, and highly significant if $\leq .001$

Table 9: Poisoning Severity Score (PSS) according to the type of exposure.

\begin{tabular}{|c|c|c|c|c|c|c|c|c|c|c|c|c|c|}
\hline \multirow{3}{*}{ Toxic agent } & \multicolumn{13}{|c|}{ Poisoning Severity Score ${ }^{a}$} \\
\hline & \multicolumn{2}{|c|}{$\begin{array}{c}0 \\
\text { None } \\
\end{array}$} & \multicolumn{2}{|c|}{$\begin{array}{c}1 \\
\text { Minor }\end{array}$} & \multicolumn{2}{|c|}{$\begin{array}{c}2 \\
\text { Moderate }\end{array}$} & \multicolumn{2}{|c|}{$\begin{array}{c}3 \\
\text { Severe } \\
\end{array}$} & \multicolumn{2}{|c|}{$\begin{array}{c}4 \\
\text { Fatal }\end{array}$} & \multicolumn{2}{|c|}{ Total } & \multirow{2}{*}{$\begin{array}{c}\text { Mean } \\
\pm \text { SD }\end{array}$} \\
\hline & $\mathbf{n}$ & $\%$ & $\mathbf{n}$ & $\%$ & $\mathbf{n}$ & $\%$ & $\mathbf{n}$ & $\%$ & $\mathbf{n}$ & $\%$ & $\mathbf{n}$ & $\%$ & \\
\hline Anticholinesterases & 0 & 0 & 11 & 39.3 & 10 & 35.7 & 6 & 21.4 & 1 & 3.6 & 28 & 100 & $1.9 \pm .9$ \\
\hline Digoxin & 0 & 0 & 6 & 35.3 & 9 & 53 & 2 & 11.8 & 0 & 0 & 17 & 100 & $1.8 \pm .7$ \\
\hline Beta blockers & 3 & 21 & 5 & 35.7 & 5 & 35.7 & 1 & 7.1 & 0 & 0 & 14 & 100 & $1.3 \pm .9$ \\
\hline $\mathrm{P}^{\mathrm{b}}$ (Kruskal-Wallis test) & & & & & & .16( & & & & & & & \\
\hline
\end{tabular}

${ }^{a}$ Poisoning Severity Score developed by the International Program on Chemical Safety (IPCS), the European Community (EC), and the European Association of Poisons Centers and Clinical Toxicologists (EAPCCT) (Persson et al., 1998).

${ }^{b} P$ is significant if $\leq .05$, and highly significant if $\leq .001$ 
Table 10: Regression analysis of extra-cardiac findings for prediction of cardiotoxicity.

\begin{tabular}{|c|c|c|c|c|}
\hline Variable & Adjusted $\mathbf{R}^{2}$ & SE & $\mathbf{P}^{\mathbf{a}}$ & Sig. \\
\hline Gender & .06 & 1 & .052 & $\mathrm{NS}$ \\
\hline Vomiting & .1 & .5 & .01 & $\mathrm{~S}$ \\
\hline Metabolic acidosis & -.11 & .5 & .007 & $\mathrm{~S}$ \\
\hline Respiratory alkalosis & .06 & .5 & .038 & $\mathrm{~S}$ \\
\hline Poison Severity Score & .27 & .4 & $<.0001$ & $\mathrm{HS}$ \\
\hline Delay & .18 & 1 & .1 & $\mathrm{NS}$ \\
\hline
\end{tabular}

${ }^{a} P$ is significant if $\leq .05$, and highly significant if $\leq .001$

Table 11: Sensitivity and specificity of PSS in diagnosis of cardiotoxicity.

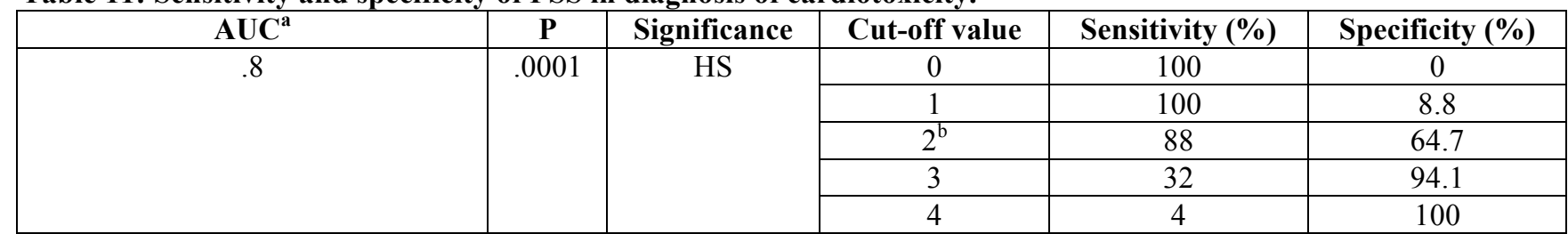

${ }^{a}$ AUC, area under the curve

${ }^{b}$ Cut-off value for a "general optimum test"

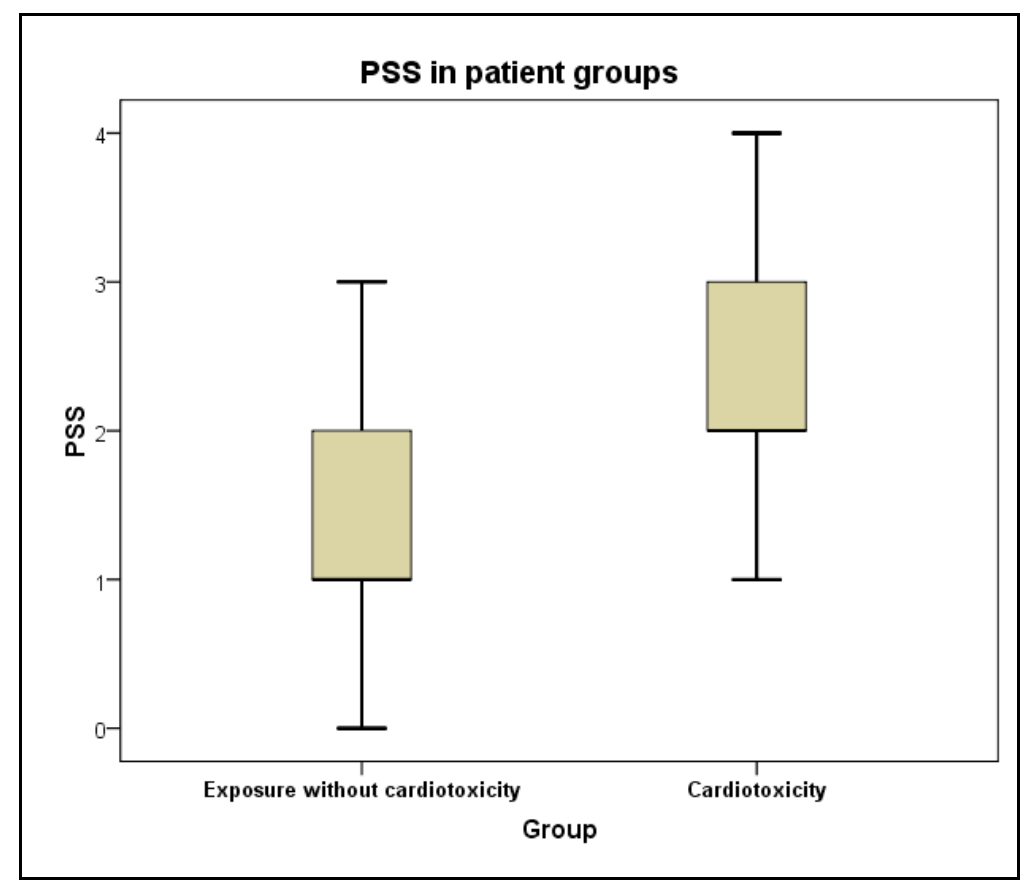

Figure 1: Box-plot showing PSS for patients under study. 


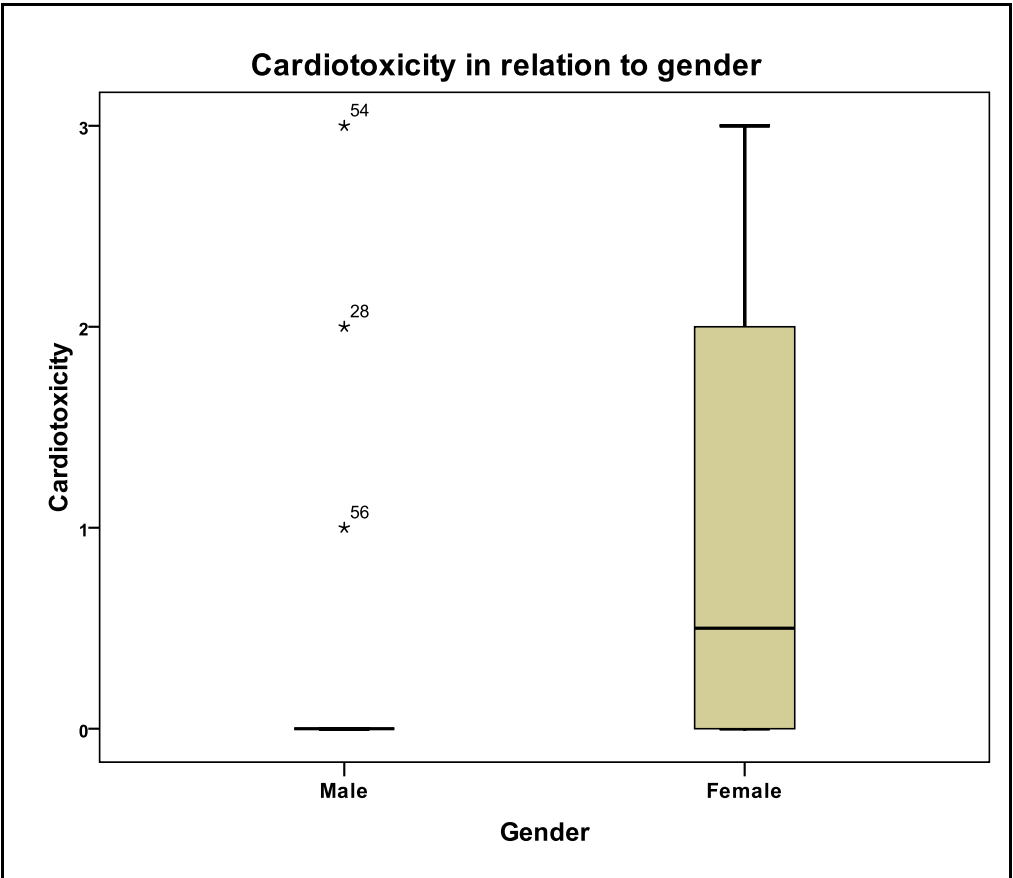

Figure 2: Box-plot correlating cardiotoxicity to gender.

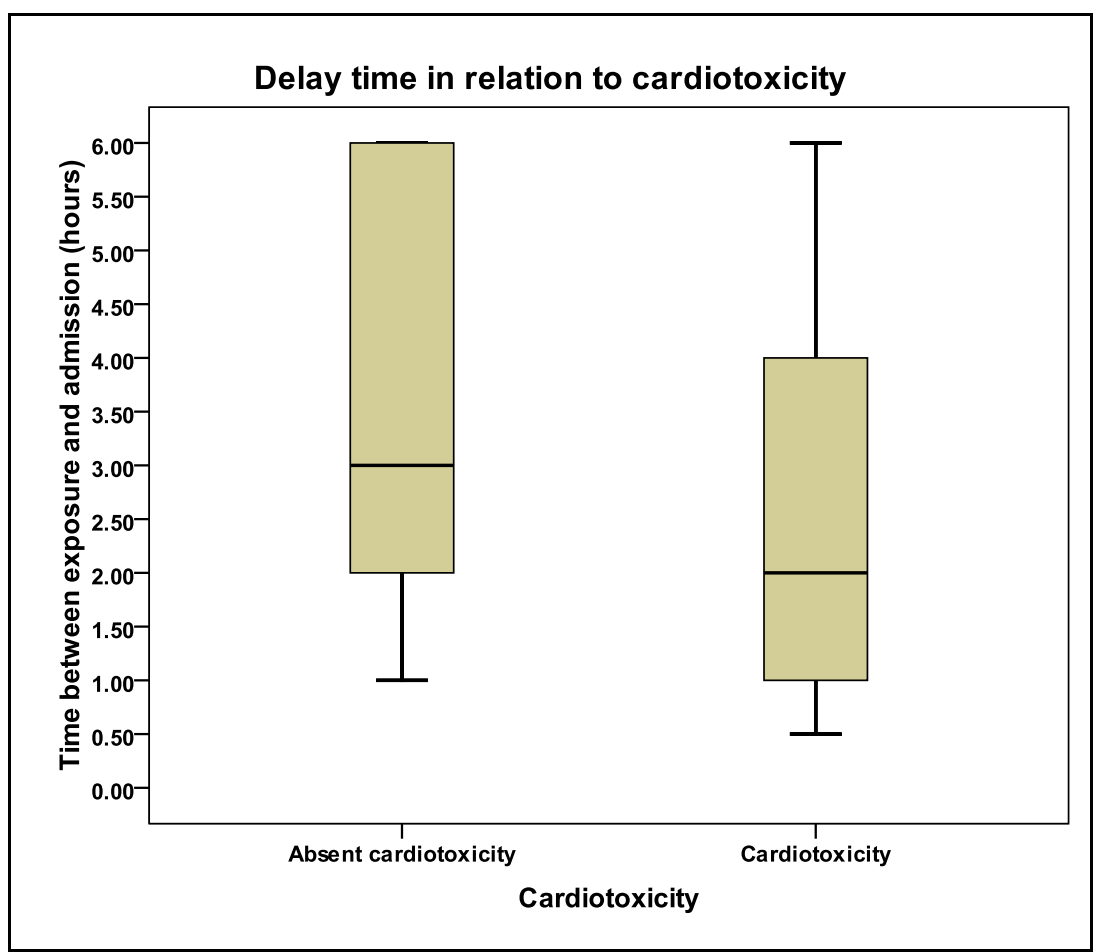

Figure 3: Box plot correlating delay time to the degree of cardiotoxicity. 


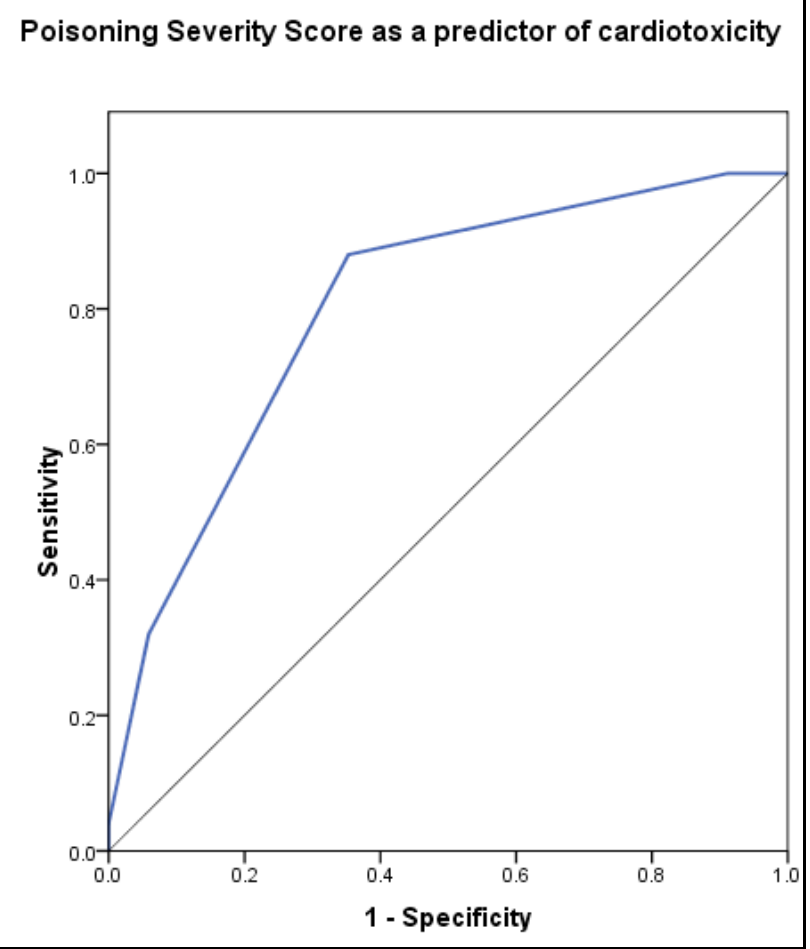

Figure 4: Receiver Operating Characteristic curve of Poison Severity Score (PSS), for the diagnosis of cardiotoxicity.

\section{Discussion}

The association between cardiotoxicity and female gender shown in this study was recognized by others. For example, female gender acted as a risk factor for anthracycline-induced cardiotoxicity (Krischer et al., 1997; Abu-Khalaf \& Harris, 2009). On the other hand, Liu et al., (2008) demonstrated no statistical differences in terms of sex between the survival and death groups in their series of OP poisoning. Similarly, Saadeh et al., (1997) and Yurumez et al., (2009) did not observe significant difference in the OP-induced ECG changes between males and females. Moreover, death risk in acute digitalis poisoning was shown to be higher in males (Dally et al., 1981). This was argued later, when digoxin therapy was revealed to be associated with increased risk of death among women, but not men (Rathore et al., 2002). As to the beta blocker overdose, although females outnumbered males in the study of Love et al., (2000), gender was not significantly correlated to beta blockerinduced cardiovascular morbidity.

In general, the association between female gender and cardiotoxicity was related to differences in oxidative stress, differential expression of drug-resistance genes, and body composition. Females have more body fat than males with the same body-surface area. At the same time, some drugs do not reach a high concentration in adipose tissue and their clearance is reduced with increased body fat. Consequently, equivalent doses could lead to higher concentrations in non-adipose tissue in females (including the heart), triggering cardiac disturbances (Lipshultz et al., 1995). On top, female sex was found to facilitate drug-induced prolongation of the
QTc (Vincent, 2000). Matyal (2008) stated that women have smaller coronary arteries, more frequent diastolic dysfunction, and shorter cardiac cycle. In addition, they are more prone to develop arrhythmias and react differently to antiarrhythmic drugs.

Concerning the lag between exposure and admission, Sam et al., (2009) found a linear correlation between it and the severity of poisoning. However, they concluded that this duration had no influence over the clinical outcome. On the contrary, less delay was associated with higher grades of cardiac affection in the present study. This highlighted what was demonstrated by Akdur et al., (2010) that pre-hospitalization period in PSS grade 2 and 4 was shorter than grade 1 and 3 . This paradoxical finding may be justified according to Goldberg et al., (2000) who speculated that more severe symptoms might encourage patients to seek care more rapidly. Confirming this is what was reported that toxicity from beta blocker exposure generally develops within 2 (Frishman et al., 1979) to $6 \mathrm{~h}$ of ingestion (Reith et al., 1996), which means that milder cases are expected to attend to hospital lately or do not attend at all. In addition, the time course for progression and resolution of cardiac glycoside-induced cardiotoxicity is variable (Roberts et al., 2005).

On the subject of clinical observations in the present analysis, the appearance of vomiting as an independent predictor of cardiotoxicity concurred with Dally et al., (1981) who declared that death rate associated with acute digitalis toxicity was significantly correlated with the presence of vomiting. As well, Pap et 
al., (2005) found that profuse vomiting was a predictive risk factor for severity and fatality form acute digoxin poisoning. Regarding anticholinesterases, Brahmi et al., (2006) stated that muscarinic and nicotinic signs give evidence on intoxication but cannot be used as prognostic factors. Although vomiting is considered a very common non-specific manifestation of acute poisoning (Mofenson, 1998), its correlation to cardiotoxicity in the current work seems logic, as some of the mechanisms underlying cardiovascular disturbances may precipitate vomiting, especially in patients exposed to anticholinesterases (acetylcholine accumulation) or digoxin (vagotonic effect). In addition, severe vomiting may be accompanied by hypotension regardless the type of toxic exposure (Pleuvry, 2006).

As to the metabolic acidosis, previous studies demonstrated that patients with cardiotoxicity commonly had acidosis more than non-cardiotoxic ones (Saadeh et al., 1997; Karki et al., 2004; Mohammed et al., 2007), and that was in accord with the present study showing metabolic acidosis as an independent predictor of cardiotoxicity. Authors have reported that OP poisoning contributes to a variety of cardiac and ECG manifestations and may result in hypotension or hypoperfusion. Such results might be the main contribution that led to the metabolic acidosis in acute OP poisoning (Karki et al., 2004; Liu et al., 2008). In digoxin toxicity, metabolic acidosis can ensue as a result of hemodynamic instability related to the presence of a dysrhythmia, CHF, or dehydration secondary to severe vomiting and decreased oral intake (TOXBASE, 2008; Patel \& James, 2011). This also applies for beta blockers, which can precipitate metabolic acidosis by inducing hypotension as a result of bradycardia and negative inotropy (Toda, 2003). The aforementioned studies showed that metabolic acidosis originates from- and contributes to cardiovascular derangement, thus its correlation to cardiotoxicity is explicit.

As with metabolic acidosis, respiratory alkalosis was an independent predicator of cardiotoxicity. In toxicology, respiratory alkalosis can occur as a result of hyperventilation associated with anxiety, fear, hysteria, response to pain, with metabolic acidosis, and excessive mechanical ventilation (Messina, 2011), in addition to direct CNS stimulation and fever (Mofenson, 1998). It can also result from hyperventilation induced by hypothermia (Mofenson, 1998; Vassallo \& Delaney, 2006), which may occur due to CNS depression, hypoglycemia, cutaneous vasodilatation, and hemodynamic instability (Mofenson, 1998; Edelstein et al., 2011). The significantly higher incidence of respiratory alkalosis in cardiotoxicity group compared to non-cardiotoxic group may be justified by severer anxiety, pain, and hemodynamic instability. In addition, respiratory alkalosis and associated hypocapnia shift the hemoglobin-oxygen dissociation curve leftward leading to tissue hypoxia with subsequent cardiac rhythm disturbances and myocardial depression (DuBose, 2005).

The highly significant positive correlation noticed between the length of ICU/total hospital stay and degree of cardiotoxicity denotes that severer cardiotoxicity was associated with longer ICU and total hospital stay time. These findings were in agreement with Rehiman et al., (2008) who demonstrated significant correlation between the average duration of hospital stay and the severity of poisoning. Yet, they found no statistically significant correlation between length of IP stay and the severity of cardiotoxicity. Also, Roberts et al., (2005) noticed that median length of hospital stay was significantly prolonged in severe anticholinesterase poisonings compared with milder cases. The correlation of ICU stay to the severity of cardiotoxicity in the present analysis was not unexpected. Poisoning-induced cardiovascular disturbances are among major indications of ICU admission. In addition, other factors that can influence ICU admission decisions include treatment and specific patient characteristics (Kirk \& Pope, 2006). Absence of correlation between IP stay and cardiotoxicity may be because most of IP cases were either mild on admission or recovered cases discharged from ICU.

As to the PSS, it incorporates clinical and ECG observations, in addition to the basic laboratory findings, such as ABG, electrolytes, and blood glucose. In addition, this evaluation scale would provide rapid triage and objective status assessment. All these advantages make PSS particularly useful in health care facilities that lack advanced analytical assistance (Sam et al., 2009). Additionally, the present study recognized PSS as an independent predictor of cardiotoxicity. A PSS of 2 has a sensitivity of $88 \%$ and a specificity of $64.7 \%$ for prediction and early diagnosis of cardiotoxicity. These findings compared well with those published by Casey et al., (1998), who inferred that PSS is helpful in assessing the clinical severity, the likelihood of further deterioration, the selection of cases warranting follow-up, and the need for referral to a clinical toxicologist. In their study, Abd El Salam et al., (2011) used PSS on admission for initial grading of 100 patients acutely exposed to hydrocarbon in Alexandria. The results showed the ability of the score to predict outcome including mortality in ICU patients. They also substantiated that patients with a PSS $\geq 2$ could be directly admitted to the ICU for possible need of mechanical ventilation. Statistically significant positive correlations were also concluded between PSS and the severity and outcome of toxicity by paracetamol (Ciszowski et al., 2005) and carbon monoxide (Cevik et al., 2006).

In OP poisoning, Davies et al., (2008) stated that PSS on admission was able to predict death. They concluded that a PSS of 3 has a sensitivity of $66 \%$ and specificity of $88 \%$ for predicting death and score of 2 or more has sensitivity and specificity of $78 \%$ and $79 \%$, respectively. In addition, Akdur et al., (2010) suggested PSS as an effective tool for determination of the severity of OP poisoning. In another study, Sam et al., (2009) noticed that patients who had low GCS showed higher 
PSS. They also announced a statistically significant correlation between the PSS grades and the need for ventilation, prehospitalization period, IMS, and mortality.

Regarding digoxin toxicity, PSS was correlated to profuse vomiting, hyperkalemia, bradycardia, and age over 65 years associated with primary disease (Pap et al., 2005). To the best of our knowledge, there are no published studies assessing possible correlation between PSS and beta blocker poisoning up till now.

\section{Conclusion}

Female gender, lag between exposure and admission, length of ICU stay, and total length of hospital stay were significantly correlated to the severity of cardiotoxicity. Vomiting, metabolic acidosis, alkalosis, and PSS were independent predictors of cardiotoxicity. A PSS of $2 \mathrm{had}$ a sensitivity of $88 \%$ and a specificity of $64.7 \%$ in predicating cardiotoxicity, thus implication of PSS in prediction and early diagnosis of cardiotoxicity is easy, available, and reliable, whatever the type of toxic exposure.

\section{References}

Abd El Salam, H. F., Fayed, A. M., \& Abdel Muneum, M. M. (2011). Prediction of the Outcome of Patients with Acute Hydrocarbons Poisoning using Poison Severity Scoring System; A Prospective Study. Journal of American Science , 7 (4), 509-18.

Abdel-Salam, M. E., Rezk, N. N., \& Hafez, R. N. (2005). Evaluation of Self-Poisoned Patients in Poison Control Centre of Ain Shams University As Regards Poisons, Age and Sex (Prospective Study). Ain Shams J Forensic Med Clin Toxicol , 4, 212-26.

Abu-Khalaf, M., \& Harris, L. (2009). Anthracyclineinduced cardiotoxicity: risk assessment and management. Oncology (Williston Park) , 23 (3), 239, 244, 252.

Akdur, O., Durukan, P., Ozkan, S. et al., (2010). Poisoning severity score, Glasgow coma scale, corrected QT interval in acute organophosphate poisoning. Hum Exp Toxicol, 29 (5), 419-25.

Brahmi, N., Mokline, A., Kouraichi, N. et al., (2006). Prognostic value of human erythrocyte acetyl cholinesterase in acute organophosphate poisoning. Am J Emerg Med, 24 (7), 822-7.

Casey, P. B., Dexter, E. M., Michell, J. et al., (1998). The prospective value of the IPCS/EC/EAPCCT poisoning severity score in cases of poisoning. $J$ Toxicol Clin Toxicol, 36 (3), 215-7.

Cevik, A. A., Unluoglu, I., Yanturali, S. et al., (2006). Interrelation between the Poisoning Severity Score, carboxyhaemoglobin levels and inhospital clinical course of carbon monoxide poisoning. Int J Clin Pract , 60 (12), 1558-64.
Ciszowski, K., Gomółka, E., \& Jenner, B. (2005). The influence of the dose, time since ingestion and concentration of the xenobiotic on the clinical state and severity of liver damage with patients intoxicated with paracetamol. Przegl Lek, 62 (6), 456-61.

Dally, S., Alperovitch, A., Lagier, G. et al., (1981). Prognostic factors in acute digitalis poisoning. Nouv Presse Med , 10 (27), 2257-60.

Davies, J. O., Eddleston, M., \& Buckley, N. A. (2008). Predicting outcome in acute organophosphorus poisoning with a poison severity score or the Glasgow coma scale. QJM , 101 (5), 371-9.

DuBose, T. D. (2005). Acidosis and Alkalosis. In D. L. Kasper, E. Braunwald, A. S. Fauci, S. 1. Hauser, D. L. Longo, \& J. L. Jameson (Eds.), Harrison's Principles of Internal Medicine (16th ed., pp. 270-1). New York: McGraw-Hill.

Ebrahim, W. G., El-Meligui, M. M., El Taftazani, E. A. et al., (2011). Acute Poisoning in the Elderly: Cross-Sectional Study in the Poison Control Center, Ain Shams University. Cairo: Ain Shams University.

Eddleston, M., Eyer, P., Worek, F. et al., (2005). Differences between organophosphorus insecticides in human self-poisoning: a prospective cohort study. Lancet , 366 (9495), 1452-9.

Edelstein, J. A., Li, J., Silverberg, M. A. et al., (2011). Hypothermia. (R. Kulkarni, D. Danzl, F. Talavera, J. S. Walker, \& J. D. Halamka, Editors) Retrieved Feb 5, 2012, from Medscape Reference - Emergency Medicine: http://emedicine.medscape.com/article/770542overview

Frishman, W., Silverman, R., Strom, J. et al., (1979). Clinical pharmacology of the new betaadrenergic blocking drugs. Part 4. Adverse effects. Choosing a beta-adrenoreceptor blocker. Am Heart J , 98 (2), 256-62.

Gamaluddin, H. A. (2005). Evaluation of the Magnitude of Acute Poisoning Problem Through Analysis of Cases Received at PCCA During the Year of 2004. Ain Shams University, Poison Control Center, Cairo.

Gamaluddin, H. A. (2007). Evaluation of the Magnitude of Acute Poisoning Problem through Analysis of Cases Received at PCCA during the Year of 2006. International Conference of Environmental Toxicology. Hurgada.

Gamaluddin, H. A., El Seddawy, A. H., Sakr, M. L. et al., (2006). Evaluation of Cases of Acute Poisoning Received at the Poison Control Center of Ain Shams University Hospitals (PCCA) during the Year 2005. Ain Shams $J$ Forensic Med Clin Toxicol, 6, 13-24.

Garth, D. (2010). Hypokalemia in Emergency Medicine. (E. D. Schraga, R. R. Hemphill, F. Talavera, H. A. Bessen, \& J. D. Halamka, Editors) Retrieved 
Jan 21, 2012, from Medscape Reference Emergency Medicine: http://emedicine.medscape.com/article/767448overview

Goldberg, R. J., Yarzebski, J., Lessard, D. et al., (2000). Decade-long trends and factors associated with time to hospital presentation in patients with acute myocardial infarction: the Worcester Heart Attack study. Arch Intern Med , 160 (21), 3217-23.

Goldberger, A. L. (2006). Clinical Electrocardiography: A Simplified Approach (7th ed.). St. Louis, USA: Mosby Elsevier.

Grais, I. M. (2010). Bedside skills: a 50-year personal retrospective. Tex Heart Inst J , 37 (6), 629-32.

Hack, J. B., \& Lewin, N. A. (2006). Cardioactive Steroids. In N. E. Flomenbaum, L. R. Goldfrank, R. S. Hoffman, M. A. Howland, N. A. Lewin, \& L. S. Nelson (Eds.), Goldfrank's Toxicologic Emergencies (8th ed., pp. 971-988). New York: McGraw-Hill.

Hartman, M. E., \& Cheifetz, I. M. (2011). Pediatric Emergencies and Resuscitation. In R. M. Kliegman, B. F. Stanton, J. W. St. Geme III, N. F. Schor, \& R. E. Behrman (Eds.), Nelson Textbook of Pediatrics (19th ed.). Philadelphia: Elsevier.

Hessler, R. A. (2006). Cardiovascular principles. In N. E. Flomenbaum, L. R. Goldfrank, R. S. Hoffman, M. A. Howland, N. A. Lewin, \& L. S. Nelson, Goldfrank's Toxicologic Emergencies (8th ed., pp. 364-79). New York: McGraw-Hill.

Hoefman, E., Boer, K. R., van Weert, H. C. et al., (2007). Predictive value of history taking and physical examination in diagnosing arrhythmias in general practice. Fam Pract, 24 (6), 636-41.

Jones, A. E., Aborn, L. S., \& Kline, J. A. (2004). Severity of emergency department hypotension predicts adverse hospital outcome. Shock, 22 (5), 410-4.

Kang, Y. J. (2008). Toxic Responses of the Heart and Vascular System. In C. D. Klaassen (Ed.), Casarett and Doull's Toxicology: the Basic Science of Poisons (8th ed., pp. 699-740). New York: McGraw-Hill.

Karki, P., Ansari, J. A., Bhandary, S. et al., (2004). Cardiac and electrocardiographical manifestations of acute organophosphate poisoning. Singapore Med J , 45 (8), 385-9.

Kirk, M. A., \& Pope, J. S. (2006). Use of the Intensive Care Unit. In N. E. Flomenbaum, L. R. Goldfrank, R. S. Hoffman, M. Howland, N. A. Lewin, \& L. S. Nelson (Eds.), Goldfrank's Toxicologic Emergencies (8th ed.). New York: McGraw-Hill.

Krischer, J., Epstein, S., Cuthbertson, D. et al., (1997). Clinical cardiotoxicity following anthracycline treatment for childhood cancer: the Pediatric
Oncology Group experience. J Clin Oncol, 15 (4), 1544-52.

Lipshultz, S., Lipsitz, S., Mone, S. et al., (1995). Female sex and drug dose as risk factors for late cardiotoxic effects of doxorubicin therapy for childhood cancer. N Engl J Med, 332 (26), 1738-43.

Liu, J. H., Chou, C. Y., Liu, Y. L. et al., (2008). Acidbase interpretation can be the predictor of outcome among patients with acute organophosphate poisoning before hospitalization. Am J Emerg Med , 26 (1), 2430.

Love, J. N., Howell, J. M., Litovitz, T. L. et al., (2000). Acute beta blocker overdose: factors associated with the development of cardiovascular morbidity. J Toxicol Clin Toxicol , 38 (3), 37581.

Luo, S., Michler, K., Johnston, P. et al., (2004). A comparison of commonly used QT correction formulae: the effect of heart rate on the QTc of normal ECGs. J Electrocardiol , 37 (Supp1), 8190.

Magaziner, J. L. (2007). Clinical Skills. In J. A. Flynn (Ed.), Oxford American Handbook of Clinical Medicine (1st ed.). New York: Oxford University Press.

Matyal, R. (2008). Newly appreciated pathophysiology of ischemic heart disease in women mandates changes in perioperative management: a core review. Anesth Analg, 107 (1), 37-50.

Messina, H. (2011). What Are The Causes Of Respiratory Acidosis \& Alkalosis? Retrieved Feb 5, 2012, from http://www.livestrong.com/article/200822-whatare-the-causes-of-respiratory-acidosis-alkalosis/

Mofenson, H. C. (1998). Differential diagnosis of poisonings. In P. Viccellio, T. Bania, J. Brent, R. S. Hoffman, K. W. Kulig, H. C. Mofenson, et al. (Eds.), Emergency toxicology (2nd ed., pp. 1103-216). Philadelphia: Lippincott Williams \& Wilkins.

Mohammed, E. A., Mahmmoud, S. M., El-Taftazani, E. A. et al., (2007). Evaluation of electrocardiographic changes and some laboratory investigations as predictors of prognosis of acute organophosphorus intoxicated patients. Cairo, Egypt: Faculty of Medicine, Ain Shams University.

Moore-Gillon, J. (2007). Respiratory system. In M. Swash, \& M. Glynn, Hutchison's Clinical Methods: An Integrated Approach to Clinical Practice (22nd ed., pp. 53-69). Edinburgh: Saunders Elsevier.

Pap, C., Zacher, G., \& Kárteszi, M. (2005). Prognosis in acute digitalis poisoning. Orv Hetil , 146 (11), 507-13.

Parliament, E., \& Presidency, E. (2008). Law No. 12 of 1996 promulgating the Law of the child as 
amended by Law No. 126 of 2008. Official Egyptian Journal (24).

Patel, V., \& James, P. A. (2011). Digitalis Toxicity, 12.07. (J. N. Rottman, Editor) Retrieved Jan 7, 2012, from Medscape Reference: http://emedicine.medscape.com/article/154336overview

Persson, H. E., Sjöberg, G. K., Haines, J. A. et al., (1998). Poisoning severity score. Grading of acute poisoning. J Toxicol Clin Toxicol, 36 (6), 205-13.

Pleuvry, B. J. (2006). Physiology and pharmacology of nausea and vomiting. Anaesthesia \& Intensive Care Medicine , 7 (12), 473-77.

Rathore, S. S., Wang, Y., \& Krumholz, H. M. (2002). Sex-based differences in the effect of digoxin for the treatment of heart failure. $N$ Engl J Med , 347 (18), 1403-11.

Rehiman, S., Lohani, S. P., \& Bhattarai, M. C. (2008). Correlation of serum cholinesterase level, clinical score at presentation and severity of organophosphorous poisoning. JNMA , 47 (170), 47-52.

Reith, D. M., Dawson, A. H., Epid, D. et al., (1996). Relative toxicity of beta blockers in overdose. $J$ Toxicol Clin Toxicol , 34 (3), 273-8.

Roberts, D., Fraser, J., Buckley, N. et al., (2005). Experiences of anticholinesterase pesticide poisonings in an Australian tertiary hospital. Anaesth Intensive Care , 33 (4), 469-76.

Roberts, R., Wijayaweera, K., \& Eddleston, M. (2005). Yellow Oleander Poisoning. Anuradhapura Medical Journal , 4, 12-17.

Saadeh, A. M., Farsakh, N. A., \& al-Ali, M. K. (1997). Cardiac manifestations of acute carbamate and organophosphate poisoning. Heart , 77 (5), 4614.

Sam, K. G., Kondabolu, K., Pati, D. et al., (2009). Poisoning severity score, APACHE II and GCS: effective clinical indices for estimating severity and predicting outcome of acute organophosphorus and carbamate poisoning. $J$ Forensic Leg Med , 16 (5), 239-47.

Schmitt, B. D. (2010). Vomiting without diarrhea. Retrieved Jan 14, 2012, from Packard Children's Hospital at Stanford LPCH: Northern California Children's

Hospital: http://www.lpch.org/healthLibrary/ParentCareT opics/vomiting.html

Sharma, A., Tefera, L., \& Aminzay, A. (2011). BetaBlocker Toxicity. (A. Tarabar, Editor) Retrieved Jan 20, 2012, from Medscape Reference Emergency Medicine: http://emedicine.medscape.com/article/813342overview

Simon, E. E., Hamrahian, S. M., \& Teran, F. J. (2012). Hyponatremia. (V. Batuman, J. H. Sondheimer, F. Talavera, E. Lederer, \& R. J. Schmidt, Editors) Retrieved Jan 21, 2012, from Medscape Reference - Emergency Medicine: http://emedicine.medscape.com/article/242166overview

Toda, N. (2003). Vasodilating beta-adrenoceptor blockers as cardiovascular therapeutics. Pharmacol Ther, 100 (3), 215-34.

TOXBASE. (2008). Digoxin Overdose. Retrieved Feb 5, 2012, from http://www.southend.nhs.uk/pathologyhandbook /clinical_chemistry/Guidelines/Digoxin_Overdo se.htm

Vassallo, S. U., \& Delaney, K. A. (2006). Thermoregulatory Principles. In N. E. Flomenbaum, L. R. Goldfrank, R. S. Hoffman, M. Howland, N. A. Lewin, \& L. S. Nelson (Eds.), Goldfrank's Toxicologic Emergencies (8th ed.). New York: McGraw-Hill.

Vincent, G. M. (2000). Long QT syndrome. Cardiol Clin, 18 (2), 309-25.

Yurumez, Y., Yavuz, Y., Saglam, H. et al., (2009). Electrocardiographic findings of acute organophosphate poisoning. J Emerg Med , 36 (1), 39-42.

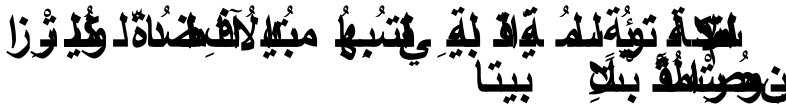

\section{و لإِيوك}
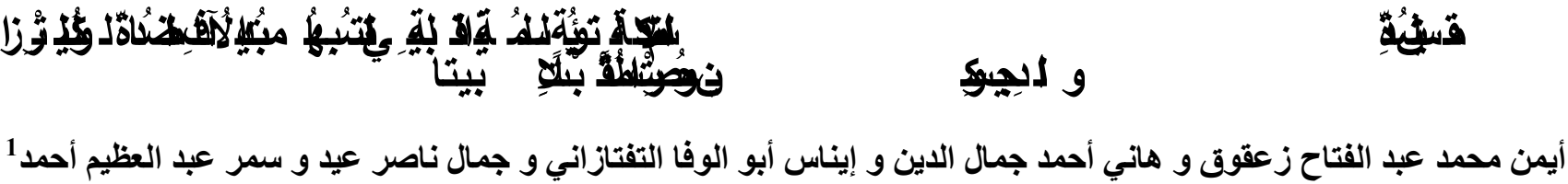

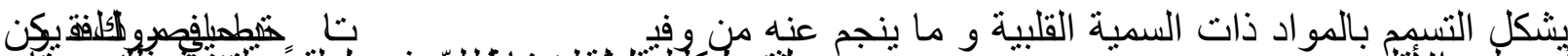

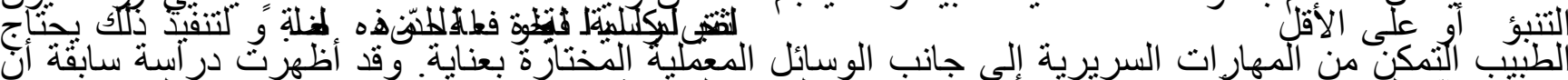

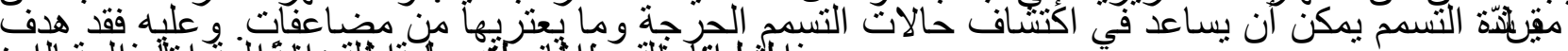

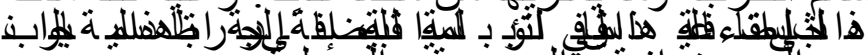

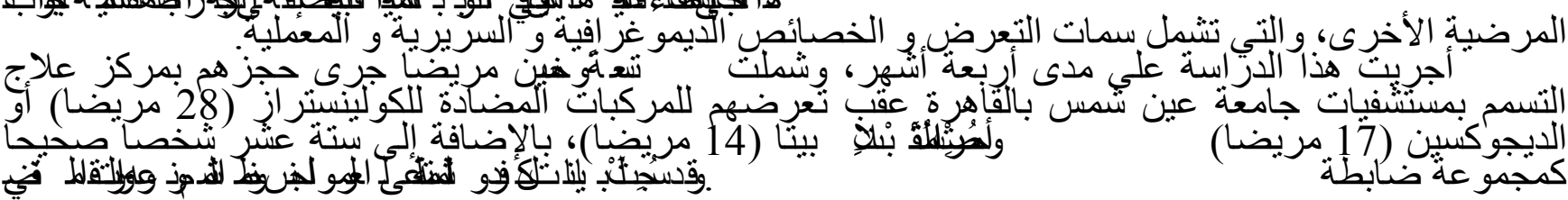




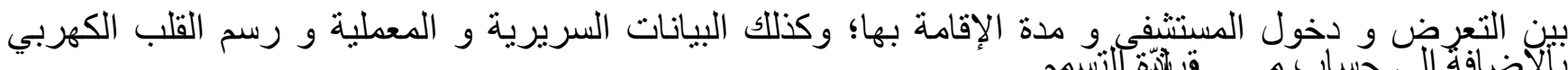

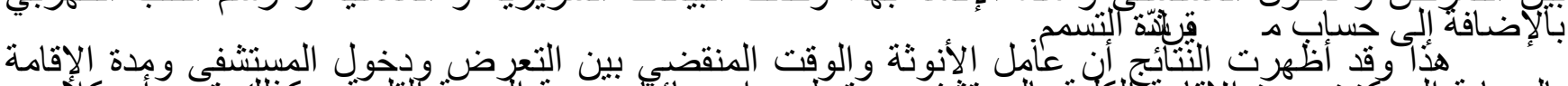

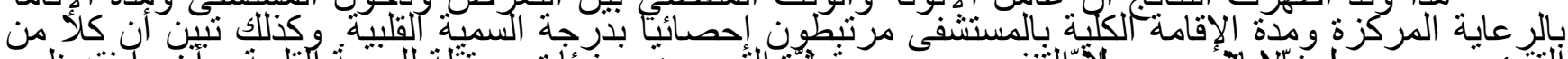

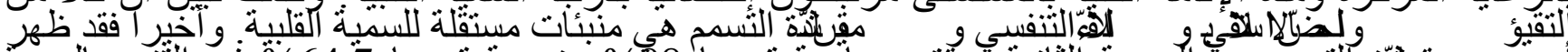

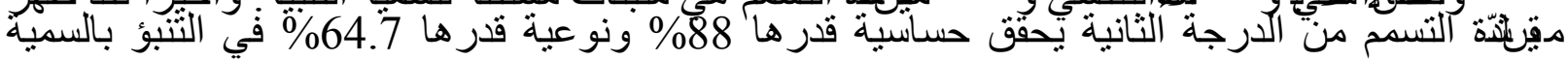

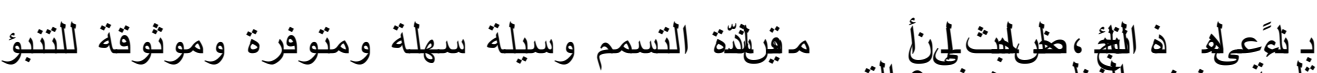

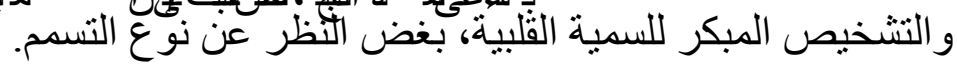
آقسم الطب الشرعي و السموم كلية الطب جامعة عين شمس 